\title{
Magnetized quark-gluon plasma at the LHC
}

\author{
V. Skalozub* \\ Oles Honchar Dnipropetrovsk National University, 49010 Dnipro, Ukraine \\ P. Minaiev ${ }^{\dagger}$ \\ Oles Honchar Dnipropetrovsk National University, 49010 Dnipro, Ukraine
}

\begin{abstract}
In QCD, the strengths of the large scale temperature dependent chromomagnetic, $B_{3}, B_{8}$, and usual magnetic, $H$, fields spontaneously generated in quark-gluon plasma after the deconfinement phase transition $(D P T)$, are estimated. The consistent at high temperature effective potential accounting for the one-loop plus daisy diagrams is used. The heavy ion collisions at the LHC and temperatures $T$ not much higher than the phase transition temperature $T_{d}$ are considered.

The critical temperature for the magnetized plasma is found to be $T_{d}(H) \sim 110-120 \mathrm{MeV}$. This is essentially lower compared to the zero field value $T_{d}(H=0) \sim 160-180 \mathrm{MeV}$ usually discussed in the literature. Due to contribution of quarks, the color magnetic fields act as the sources generating $H$. The strengths of the fields are $B_{3}(T), B_{8}(T) \sim 10^{18}-10^{19} G, H(T) \sim 10^{16}-10^{17} G$ for temperatures $T \sim 160-220 \mathrm{MeV}$. At temperatures $T<110-120 \mathrm{MeV}$ the effective potential minimum value being negative approaches to zero. This is signaling the absence of the background fields and color confinement.
\end{abstract}

\section{Introduction}

At the LHC experiments, in heavy ion collisions a new matter phase - quark-gluon plasma $(Q G P)$ - has to be produced. The deconfinement phase transition temperature is expected to be of order $T_{d} \sim 180-200 \mathrm{MeV}$. In theory, investigation of the $D P T$ and $Q G P$ properties were carried out by different method - analytic perturbative and nonperturbative, various numerical methods and Monte-Carlo simulations on a lattice (see, for example, [1] - 6]). QGP and strong magnetic fields had been existed in the hot Universe [8], 9].

One of distinguishable properties of nonabelian gauge fields at high temperature is a spontaneous vacuum magnetization. It is closely related with asymptotic freedom. In fact, asymptotic freedom at high temperature is always accompanied by the background stable, temperature dependent and long range chromo(magnetic) fields [1]. The magnetization phenomenon was investigated in detail in $S U(3)$ gluodynamics [10] and supersymmetric theories [13, 14] by analytic methods and in $S U(2)$ gluodynamics [16, [18] by the Monte-Carlo simulations on a lattice. In all these cases the spontaneous creation of magnetic fields has been detected. Within application to the early Universe the spontaneous vacuum magnetization in the electroweak sector of the standard model is described in review paper [20].

The case of experiments at the $L H C$ requires a spe- cial consideration. This is because of much lower temperature $T_{d}$ as compared to the electroweak phase transition temperature $T_{e w} \sim 100 \mathrm{GeV}$. For temperatures $T_{d}<T<T_{e w}$ the scalar field condensate, supplying particle masses, screens the magnetic field $H$, which was generated at high temperatures by the $W$ boson loops. At the same time, the color magnetic fields $B_{3}, B_{8}$ remain unscreened 20. Within this scenario the question arises: whether or not there exists a mechanism generating magnetic field $H$ in between critical temperatures $T_{d}$ and $T_{e w}$ ?

In a qualitative manner it was considered in our paper 21. Therein, in particular, we have demonstrated that magnetic field $H$ can be generated due to the vacuum polarization of quark fields by the constant color magnetic fields $B_{3}$ and $B_{8}$, existed in the $Q G P$ after the $D P T$. In the effective potential of the external fields the mixing terms of the type $\sim e H \times\left(g B_{3}\right)^{3}, \sim e H \times\left(g B_{8}\right)^{3}$, etc, where $e, g$ are electromagnetic and strong interaction couplings, present and act as the sources for $H$. The field $H$ is temperature dependent and occupying a large plasma volume as the fields $B_{3}$ and $B_{8}$.

In the present paper, we investigate in detail the creation in $Q G P$ of the magnetic fields $B_{3}, B_{8}, H$ at temperatures close to the $D P T$ and estimate the field strengths. The proper time representation is used. The one-loop plus daisy diagrams effective potential of external fields

\footnotetext{
*e-mail: Skalozubv@daad-alumni.de

$\dagger$ e-mail: Minaevp9595@gmail.com
} 
$V\left(B_{3}, B_{8}, H, T\right)$ accounting for the gluons and $u-, d-$ and $s$-quarks at finite temperature is calculated. This field configuration is stable due to the daisy diagram contributions which cancel the imaginary terms presenting in the one-loop effective potential of charged gluons $V^{(1)}\left(B_{3}, B_{8}, T\right)$. For estimation of the field strengths the asymptotic high temperature expansion, derived by Mellin's transformation technique, is applied. As corollary of these investigations we observe that strong color magnetic fields $B_{3}, B_{8}$, of the order $\sim 10^{18}-10^{19} \mathrm{G}$ and usual magnetic field $H \sim 10^{16}-10^{17} \mathrm{G}$ are generated for temperatures $T \sim 160-220 \mathrm{MeV}$. The spontaneous magnetization disappears at $T \sim 110-120 \mathrm{MeV}$. This temperatures is considered as the deconfinement temperature in the presence of the fields. It is essentially lower that the one estimated without magnetic fields.

The paper is organized as follows. In next section we adduce the one-loop effective potential of quarks $V_{q}^{(1)}\left(B_{3}, B_{8}, H, T\right)$. In sect. 3 we present the one-loop contributions of gluons $V_{g l}^{(1)}\left(B_{3}, B_{8}, T\right)$ calculated in the high temperature approximation, which is sufficient for the problem under consideration. In sect. 4 the contribution of daisy diagrams is calculated in brief and the dimensionless variables used in numeric calculations are introduced. Then in sect. 5 the values of the field strengths $B_{3}(T), B_{8}(T), H(T)$ are estimated for a number of temperatures. Discussion of the results and conclusion are given in the last section. Appendix A describes the details of calculations of the quark zero temperature effective potential. Appendix B includes information about the high temperature expansion of the one-loop effective potentials $V_{q}^{(1)}, V_{g l}^{(1)}$.

\section{Quark contributions to one- loop effective potential}

In what follows, we consider the situation when temperature of $Q G P$ is not much higher than $T_{d}$. In this case, according to [10], the color magnetic fields $B_{3}$ and $B_{8}$ are spontaneously created in the gluon sector of $Q C D$ because color symmetry is restored. On the contrary, for the temperature interval $T_{d}<T<T_{e w}$ the electroweak symmetry is broken and $S U(2)$ constituent of usual magnetic field is screened by the scalar field condensate. At temperatures $T>T_{e w}$ the spontaneous generation of this field takes place also 20. Having this picture in mind we calculate the one-loop quark effective potential $V_{q}^{(1)}\left(B_{3}, B_{8}, H, T\right)$ at the background of all three fields.

To be in correspondence with the notations of [10], [21] we present the $S U(3)_{c}$ gluon field in the form

$$
A_{\mu}^{a}=B_{\mu}^{a}+Q_{\mu}^{a},
$$

where $B_{\mu}^{a}$ is background classical field and $Q_{\mu}^{a}$ presents quantum gluons. We choice the external field potential in the form $B_{\mu}^{a}=\delta^{a 3} B_{3 \mu}+\delta^{a 8} B_{8 \mu}$, where $B_{3 \mu}=H_{3} \delta_{\mu 2} x_{1}$ and $B_{8 \mu}=H_{8} \delta_{\mu 2} x_{1}$ describe constant chromomagnetic fields directed along third axis in the Euclidean space and $a=3$ and $a=8$ in the color $S U(3)_{c}$ space, respectively. The field tensor has the components: $F_{\mu \nu}^{e x t a}=$ $\delta^{a 3} F_{3 \mu \nu}+\delta^{a 8} F_{8 \mu \nu}, \quad F_{c 12}=-F_{c 21}=H_{c}, c=3,8$. We direct usual magnetic field also along third axis and choice its potential in the form: $A_{\mu}^{e x t}=H \delta_{\mu 2} x_{1}$.

We first calculate the quark spectrum in the presence of all these fields [21]. The corresponding Dirac equation reads

$$
\left(i \gamma_{\mu} D_{\mu}+m_{f}\right) \psi^{a}=0,
$$

where $\psi^{a}$ is a quark wave function, $a$ is color index, $m_{f}$ is mass of $f$-flavor quark. The covariant derivative describes the interactions with external magnetic fields $H$ and $H_{3}, H_{8}$ :

$$
D_{\mu}=\partial_{\mu}+i q_{f}|e| A_{\mu}^{e x t}+i g\left(T^{3} B_{\mu}^{3}+T^{8} B_{\mu}^{8}\right)
$$

where $T^{3}=\frac{\lambda^{3}}{2}, T^{8}=\frac{\lambda^{8}}{2}$ are the generators of $S U(3)$ group, $\lambda^{3,8}$ are Gell-Mann matrixes. Due to the choice of the potentials we can present the quark spectrum as the sum of contributions of the following external field combinations:

$$
\begin{aligned}
\mathcal{H}_{f}^{1} & =q_{f}|e| H+g\left(\frac{H_{3}}{2}+\frac{H_{8}}{2 \sqrt{3}}\right), \\
\mathcal{H}_{f}^{2} & =q_{f}|e| H+g\left(\frac{H_{8}}{2 \sqrt{3}}-\frac{H_{3}}{2}\right), \\
\mathcal{H}_{f}^{3} & =q_{f}|e| H-g \frac{H_{8}}{\sqrt{3}} .
\end{aligned}
$$

Here, $q_{f}|e|$ is electric charge of $f$-quark. Each flavor energy spectrum is given by the known expression (see, for instant, [17] )

$$
\epsilon_{i, n, \rho, f}^{2}=m_{f}^{2}+p_{z}^{2}+(2 n+1) \mathcal{H}_{f}^{i}-\rho \mathcal{H}_{f}^{i},
$$

where $p_{z}$ is momentum along the field direction, $\rho= \pm 1$.

Vacuum energy is defined as the sum of the modes having negative energy. At finite temperature, in the imaginary time formalism for fermions, it is reduced to the summation over discrete odd imaginary energies $p_{4}=\frac{(2 l+1) \pi}{\beta}, \beta=1 / T$ is inverse temperature [3], [1]. The result yields [1]

$$
\begin{array}{r}
V_{q}^{(1)}\left(T, H_{i}\right)=\frac{1}{8 \pi^{2}} \sum_{f=1}^{6} \sum_{i=1}^{3} \sum_{l=-\infty}^{\infty}(-1)^{l} \\
\times \int_{0}^{\infty} \frac{d s}{s^{3}} \exp \left(-m_{f}^{2} s-\frac{\beta^{2} l^{2}}{4 s}\right)\left[\mathcal{H}_{f}^{i} s \operatorname{coth}\left(\mathcal{H}_{f}^{i} s\right)-1\right] .
\end{array}
$$

This is expression of interest. The term with $l=0$ is the vacuum energy $V_{q}^{(1)}\left(H_{i}\right)$. Different type asymptotic expansions of (6) are given in [21]. 


\section{Gluon contributions to one- loop effective potential}

In this section, to give a self-contained presentation, we describe in brief the one-loop contributions of gluons to the effective potential. A detailed calculations are carried out in 10. The Lagrangian of $S U(3)_{c}$ gluodynamics is well known

$$
L=-\frac{1}{4} F_{\mu \nu}^{a} F_{\mu \nu}^{a}+L_{g f}+L_{g h},
$$

where $F_{\mu \nu}^{a}=\partial_{\mu} A_{\nu}^{a}-\partial_{\nu} A_{\mu}^{a}-g f^{a b c} A_{\mu}^{b} A_{\nu}^{c}$ is the field strength tensor, $f^{a b c}$ are the group structure constants, $a=1,2, \ldots, 8$. In actual calculations, we use the decomposition of the gauge field potential as in (11). The metric is chosen to be Euclidian for introducing the imaginary time formalism. The gauge fixing term in (7) is

$$
L_{g f}=-\frac{1}{2}\left(\partial_{\mu} Q_{\mu}^{a}+g f^{a b c} B_{\mu}^{b} Q_{\nu}^{c}\right)^{2},
$$

and $L_{g h}$ represents the ghost field Lagrangian. The components $Q_{\mu}^{a}$ with $a=1,2,4,5,6,7$ correspond to the color charged gluons. In calculations it is convenient to use the "charged basis" of gluons

$$
\begin{aligned}
W_{1 \mu}^{ \pm} & =\frac{1}{\sqrt{2}}\left(Q_{\mu}^{1} \pm i Q_{\mu}^{2}\right), \\
W_{2 \mu}^{ \pm} & =\frac{1}{\sqrt{2}}\left(Q_{\mu}^{4} \pm i Q_{\mu}^{5}\right), \\
W_{3 \mu}^{ \pm} & =\frac{1}{\sqrt{2}}\left(Q_{\mu}^{6} \pm i Q_{\mu}^{7}\right) .
\end{aligned}
$$

In terms of these fields the spectrum of the charged gluons looks like the spectrum of the spin one massless charged particle with gyromagnetic ratio $\gamma=2$. That is, we have to set in (5) $\mathrm{m}=0$ and put $\rho= \pm 2$ for gluons and $\rho=0$ for ghosts. As the values of the $\mathcal{H}^{i}$ we have to put $H=0$ and use the combinations of the neutral components $H_{3}$ and $H_{8}$ entering (4).

The detailed calculation of the one-loop effective potential for $S U(2)$ was carried out in [11, [12]. It corresponds to each $S U(2)$ subgroup of the $S U(3)_{c}$ group. These subgroups are related with the components $W_{r \mu}^{ \pm}$, $r=1,2,3$ of the basis (9) and the corresponding combinations of $H_{3}$ and $H_{8}$ (for more details see [10]):

$$
\begin{aligned}
B_{r=1, \mu} & =B_{\mu}^{3}, \\
B_{r=2, \mu} & =\sqrt{\frac{3}{2}} B_{\mu}^{8}+\frac{1}{2} B_{\mu}^{3}, \\
B_{r=3, \mu} & =\sqrt{\frac{3}{2}} B_{\mu}^{8}-\frac{1}{2} B_{\mu}^{3} .
\end{aligned}
$$

Just these fields enter the covariant derivatives related with the $S U(2)$ subgroups, $D_{\mu}^{r}=\partial_{\mu}+i g B_{\mu}^{r}$.

The one-loop gluon contribution to the effective potential, presented in the form similar to ([6), reads

$$
V_{g}^{(1)}\left(T, H_{r}\right)=\frac{1}{8 \pi^{2}} \sum_{r=1}^{3} \sum_{l=-\infty}^{\infty}
$$

$\times \int_{0}^{\infty} \frac{d s}{s^{2}} e^{-i \mu^{2} s} \exp \left(i l^{2} \beta^{2} / 4 s\right)\left[\frac{g H_{r} \cos \left(2 g H_{r} s\right)}{\sin \left(g H_{r} s\right)}-\frac{1}{s}\right]$,

where $\mu^{2}-i \epsilon, \epsilon \rightarrow 0$ is a parameter playing the role of the normalization point in the field. It is useful for analytic continuations from the weak fields $g H_{r} \leq \mu^{2}$ to the fields $g H_{r} \geq \mu^{2}$ when an imaginary part of the effective potential is calculated. The term with $l=0$ gives the zero temperature (vacuum) part and other terms describe the statistical part.

In what follows, we take into consideration the gluon contributions in the high temperature limit $T>>$ $\left(g H_{r}\right)^{1 / 2} \geq \mu$, which is sufficient for our problem. The calculation of $V_{g}^{(1)}\left(T, H_{r}\right)$ for this case was carried out by Mellin's transformation technique described in Appendix B for the quark effective potential (6). For gluons it is presented in [10, Eq. (10) :

$$
\begin{gathered}
V_{g}^{a s}\left(T, H_{3}, H_{8}\right)=\frac{H_{3}^{2}}{2}+\frac{11}{32} \frac{g^{2}}{\pi^{2}} H_{3}^{2} \log \left[\frac{T}{\mu}\right] \\
-\left(g H_{3}\right)^{3 / 2} \frac{T}{3 \pi}+\frac{H_{8}^{2}}{2}+\frac{11}{16} \frac{g^{2}}{\pi^{2}} H_{8}^{2} \log \left[\frac{T}{\mu}\right] \\
-\left(\lambda_{+}^{3 / 2}+\left|\lambda_{-}\right|^{3 / 2}\right)\left(\frac{3}{2}\right)^{3 / 4}\left(g H_{8}\right)^{3 / 2} \frac{T}{3 \pi} \\
-i\left[\left(g H_{3}\right)^{3 / 2}+\left(\lambda_{+}^{3 / 2}+\left|\lambda_{-}\right|^{3 / 2}\right)\left(\frac{3}{2}\right)^{3 / 4}\left(g H_{8}\right)^{3 / 2}\right] \frac{T}{2 \pi} \\
+O\left(g^{2} H_{3,8}^{2}\right),
\end{gathered}
$$

where tree-level terms of $H_{3}$ and $H_{8}$ were added, $\lambda_{ \pm}=1 \pm \frac{1}{\sqrt{6}} \frac{H_{3}}{H_{8}}$ and $\mu$ is normalization point. This expression contains the imaginary part related with the lower state of the gluon spectrum $\epsilon_{n=0, \rho=2}^{2}=p_{z}^{2}-g H_{r}$. It was realized already, this term is exactly canceled by the imaginary term coming from the daisy diagrams for charged gluons [12, 10]. This is the main reason for using this approximation in further analysis.

\section{Contribution of daisy diagrams}

As it is well known [15], at finite temperature along with a one-loop effective potential we have to take into consideration the so-called daisy diagram contributions which account for the long distance correlations. Graphically, this is a series of one-loop gluon diagrams with infinite number of insertions of the polarization tensors taken at zero external momenta, $\Pi\left(T, g, H_{3}, H_{8}\right)$. They have the order $\sim g^{3 / 2}$ in coupling constant and therefore must 
be included in the effective potential after the one-loop terms. The two-loop contributions have the order $\sim g^{2}$. This order terms were neglected in (12).

The calculations of the daisy diagram contributions coming from charged gluons are described in [12, 10. In notation (10) the part given by the unstable modes is

$$
\begin{aligned}
& V_{\text {unst. }}^{\text {daisy }}=\frac{T}{2 \pi} \sum_{r=1}^{3}\left(g H_{r}\right)\left[\Pi^{r}\left(H_{r}, T\right)-g H_{r}\right]^{1 / 2} \\
& +i\left[\left(g H_{3}\right)^{3 / 2}+\left(\lambda_{+}^{3 / 2}+\left|\lambda_{-}\right|^{3 / 2}\right)\left(\frac{3}{2}\right)^{3 / 4}\left(g H_{8}\right)^{3 / 2}\right] \frac{T}{2 \pi} .
\end{aligned}
$$

Here, $\Pi^{r}\left(H_{r}, T\right)$ denotes the one-loop polarization tensors of color charged gluons averaged over the ground (unstable) state of the tree-level spectrum taken at $p_{3}=$ $0: \mid n=0, \rho=2>$. As we see, the imaginary parts in (12) is exactly canceled by the one in (13). Thus, the effective potential $V_{g}^{(1) a s}+V_{\text {unst. }}^{\text {daisy }}$ is real if $\left[\Pi^{r}\left(H_{r}, T\right)-g H_{r}\right]>0$.

Detailed calculations of the charged gluon polarization tensor have been carried out in [10] (see also review [20]). The most important for us is the temperature and field dependencies of it: $\Pi^{r}(H, T) \sim g^{2} \sqrt{g H} T$. Hence, the remaining after the cancelations part in the effective potential is of the order $\sim g^{2} g^{1 / 4}$. It is smaller than the accuracy preserved in (12). We can conclude that the role of the unstable mode daisies consists in the stabilization of the effective potential in the chosen approximation in coupling $g$. All other terms are negligibly small and have to be dropped. As concerns the contributions of the neutral gauge field daisies, they have the order $\sim g^{5 / 2}$ and have to be dropped also 10. Thus, the consistent effective potential for gluons including the one-loop plus daisies is real.

To carry out numeric calculations we use the dimensionless variables for the effective potential, temperature and fields. We consider the proton mass $m_{p}=938.27208$ $\mathrm{MeV}$ as a reference parameter and introduce the dimensionless variables:

$$
\begin{gathered}
V_{q, g}^{0}=\frac{V_{q, g}^{(0)}}{m_{p}^{4}}, V_{q, g}^{T}=\frac{V_{q, g}^{(T)}}{m_{p}^{4}}, \mu_{f}=\frac{m_{f}}{m_{p}}, \\
h_{f, a}=\frac{\mathcal{H}_{f}^{a}}{m_{p}^{2}}, \beta_{p}=m_{p} \beta ; \omega_{f}=\mu_{f} \beta_{p} .
\end{gathered}
$$

We also take into consideration three sorts of quarks with the masses and electric charges

$$
\begin{gathered}
m_{u}=336 \mathrm{MeV}, q_{u}=\frac{2}{3}|e|, \\
m_{d}=340 \mathrm{MeV}, q_{d}=-\frac{1}{3}|e|, \\
m_{s}=486 \mathrm{MeV}, q_{s}=-\frac{1}{3}|e|
\end{gathered}
$$

and the coupling values $\alpha_{s}=1, \alpha_{e}=\frac{1}{137}, g=\sqrt{4 \pi}$, $|e|=\sqrt{\frac{4 \pi}{137}}$. The dimensionless field strengths are: $x=\frac{|e| H}{m_{p}^{2}}, x_{3}=\frac{g H_{3}}{m_{p}^{2}}, x_{8}=\frac{g H_{8}}{m_{p}^{2}}$. The field combinations in (44) and (10) should be expressed in term of them. In next section we present the results of the calculations fulfilled for a number of temperatures. Details of calculations are placed in the Appendices.

\section{$5 \quad$ Estimate of the field strengths}

The total effective potential used in our investigation consists of the one-loop quark contribution (6) including the zero temperature term $V_{q}^{0}$ with $\mathrm{l}=0$ and the statistical part $V_{q}^{T}$ with $l \neq 0$ and the gluon contributions (12), (13) presented above. The calculation of the zero temperature quark potential is given in Appendix A. To be in correspondence with the gluon sector approximation, we apply the high temperature expansion for quark sector also. The calculations are given in Appendix B. The final expressions read

$$
\begin{array}{r}
V_{q}^{0}=\frac{x^{2}}{2 e^{2}}+\frac{x_{3}^{2}}{2 g^{2}}+\frac{x_{8}^{2}}{2 g^{2}}+\frac{1}{8 \pi^{2}} \sum_{f} \sum_{a=1}^{3}\left[\frac{1}{3} h_{f, a}^{2}\right. \\
-2 h_{f, a}^{2}\left(2 \Gamma_{1}\left(\frac{\mu_{f}^{2}}{2 h_{f, a}}\right)+\frac{\mu_{f}^{2}}{2 h_{f, a}} \ln \frac{\mu_{f}^{2}}{2 h_{f, a}}+2 \zeta^{\prime}(-1)\right) \\
\left.+\frac{1}{3} h_{f, a}^{2} \ln \frac{\mu_{f}^{2}}{2 h_{f, a}}+\frac{1}{2} \mu_{f}^{4} \ln \frac{\mu_{f}^{2}}{2 h_{f, a}}-\frac{1}{4} \mu_{f}^{4}\right]
\end{array}
$$

and

$$
\begin{array}{r}
V_{q}^{T, a s}=\frac{1}{4 \pi^{2}} \sum_{f} \sum_{a=1}^{3}\left[\frac{2}{3} h_{f, a}^{2}\right. \\
\times\left[\frac{1}{2}\left(\gamma+\ln \left(\frac{\omega_{f}}{\pi}\right)\right)+\frac{7 \zeta^{\prime}(-2)}{4} \omega_{f}^{2}+\frac{31 \zeta^{\prime}(-4)}{64} \omega_{f}^{4}\right] \\
\left.-\frac{h_{f, a}^{4}}{90 \mu_{f}^{4}}\left[-1+\frac{31 \zeta^{\prime}(-4)}{8} \omega_{f}^{4}\right]\right] .
\end{array}
$$

Here, $\Gamma_{1}(x)$ is generalized Gamma function, $\zeta(x)$ is $\zeta$ function, the notations are given in (14).

These two expressions plus (12) and (13) are used in the estimation of the magnetic field strengths. For doing so we numerically solve the stationary equations

$$
\frac{\partial V\left(H, H_{3}, H_{8}, T\right)}{\partial H}=0, \frac{\partial V\left(H, H_{3}, H_{8}, T\right)}{\partial H_{3,8}}=0
$$

at a number of fixed temperatures and obtain the roots $h_{m i n}^{i}(T)$. If for a particular set of $h_{m i n}^{i}(T)$ the total effective potential is negative, we have to conclude that these magnetic fields are spontaneously generated. The results of the calculations are presented in Table 1. 


\begin{tabular}{|c|c|c|c|c|c|c|c|}
\hline $\mathrm{T}, \mathrm{MeV}$ & $x_{8}$ & $x_{3}$ & $x 10^{-3}$ & $V$ & $H_{8} 10^{19} G$ & $H_{3} 10^{18} G$ & $H 10^{17} G$ \\
\hline 120 & 0.463423 & 0.0762514 & -0.0512118 & -0.0023921 & 0.589129 & 0.96935 & -0.0762115 \\
140 & 0.783271 & 0.131652 & 0.0404301 & -0.00625276 & 0.995738 & 1.67363 & 0.0601666 \\
160 & 0.900931 & 0.15203 & 0.248859 & -0.011033 & 1.14531 & 1.93269 & 0.370343 \\
180 & 0.998792 & 0.168842 & 0.476241 & -0.0167341 & 1.26972 & 2.14641 & 0.708724 \\
200 & 1.09212 & 0.184812 & 0.727744 & -0.0235134 & 1.38836 & 2.34943 & 1.083 \\
220 & 1.18195 & 0.200116 & 1.00549 & -0.0314384 & 1.50256 & 2.54399 & 1.49633 \\
\hline
\end{tabular}

Table 1.The values of the field strengths spontaneously generated at chosen plasma temperatures

In Table 1, in first column we show the temperature. The next tree columns give the values of the dimensionless field strengths, the next one shows the behavior of the dimensionless effective potential. The field strengths [Gauss] are shown in the last three columns.

We have detected the negative values of the effective potential for the stationary field strengths. It means that magnetic $H$ and chromomagnetic $H_{3}, H_{8}$ fields have to be generated spontaneously after the $D P T$ in $Q G P$. If the temperature is lower than $110-120 \mathrm{MeV}$, the effective potential value is close to zero. Hence, within the high temperature approximation adopted, we expect the background fields disappear and confinement is realized. We see from Table 1 that the strength of the magnetic field is two orders of magnitude less than the strength of the colored fields and equals $\sim 10{ }^{16} \mathrm{G}$ at the $L H C$ experiment temperatures.

\section{Discussion and conclusions}

The most interesting observation of the above investigation is two fold. Firstly, with temperature lowering the magnetic field strengths are decreased. Secondly, simultaneously the value of the effective potential in the minimum, being negative, increases and tends to zero. Beginning from the value $V_{\min } \sim-0.02$ at $T=200 \mathrm{MeV}$ it equals to -0.002 at $T=120 \mathrm{MeV}$, that is it increases in one order. Such type behavior detects that the magnetic fields act to decrease the $D P T$ temperature $T_{d}$. Magnetized $Q G P$ must be created at essentially lower temperature as compared to the zero field case.

The $T_{d}$ lowering has also been observed already in 23], 24, 25] for the DPT in applied external magnetic fields. In [24], in particular, it was found in lattice calculations that the temperature $T_{d}$ can even be reduced to zero for sufficiently strong color magnetic fields. In contrast, here we determined similar behavior for the magnetic fields spontaneously created in $Q G P$. Hence, the $D P T$ has to happen at essentially lower temperatures $\sim 110-120 \mathrm{MeV}$. For these temperatures the minimum value of the total effective potential is very close to zero. In the used approach this means the magnetic field screening and color comfinement. Really, as we noted already, asymptotic freedom at high temperature has always be accompanied by temperature dependent background magnetic fields [11. Screening of these fields re- flects the destroying of the asymptotic freedom regime and color comfinement at low temperature. The spontaneously generated temperature dependent macroscopic magnetic fields are intrinsic constituent of $Q G P$ and the signals of the DPT. In short, deconfinement is always accompanied by macroscopic long range chromo(magnetic) fields.

Above we have applied the approximation for the effective potential including the one-loop plus daisies, which is real in the leading order $\sim O\left(g^{3 / 2}\right)$ in coupling constant. Here, we mention a number of the mechanisms for the magnetic field stabilization at finite temperature. In detail this problem was investigated by either analytic methods of field theory or simulations on a lattice. It is discussed in [20]. Qualitatively, two factors act to stabilize vacuum. First is a so-called $A_{0}$ condensate related with the Polyakov loop 22 which appears after the $D P T$. It enters the gluon spectrum of the type (5) in the form $\cdots+\left(g A_{0}\right)^{2}$ and acts in favor of eliminating the instability. Second are the radiation corrections forming the magnetic mass of charged gluons $\Pi_{c h}^{\perp}(H, T) \sim g^{2} \sqrt{g H} T$ and having a large positive real part [12, which also acts to stabilize vacuum. These mechanisms have been proven also in lattice simulations [16, [18. So, we stress again that the used approximation for the effective potential is consistent and reliable.

Now, let us compare the obtained results with that of in 21] where to clarify the role of the quark loop effects the color magnetic fields $H_{3}(T)$ and $H_{8}(T)$ were estimated from the effective potential of the gluon fields, only. The comparison shown that the field strength $H(T)$ is $\sim 15$ per cent less in this approximation. For example, at $T=200 \mathrm{MeV} x(h)=6.2410^{-4}$ whereas from the present results we obtained $x(h)=7.2810^{-4}$. But qualitatively this is close.

As it follows from the obtained results, in $Q G P$ strong chromo(magnetic) fields of the order $H_{3,8} \sim$ $10^{18}-10^{19} \mathrm{G}$ and $H \sim 10^{16}-10^{17} \mathrm{G}$ must be present. This influences all the processes happening and may serve as the distinguishable signals of the DPT. Due to magnetization, in particular, all the initial states of charged particles have to be discrete ones. This could modify the cross sections of particular processes and detected in experiments. Moreover, the fields, as well as the $A_{0}$ condensate, generate new type processes with $C$ parity violation, which also could be the signals of the 
plasma formation. Detailed consideration of these problems will be reported elsewhere.

\section{Appendix A}

The term with $l=0$ in (6) is the vacuum energy $V_{q \cdot v a c}^{(1)}\left(H, H_{3}, H_{8}\right)$. The well known expression for it reads 17

$$
\begin{gathered}
8 \pi^{2} V_{q \cdot v a c, i, f}^{(1)}\left(H, H_{3}, H_{8}\right)= \\
=\int_{0}^{\infty} \frac{d s}{s^{3}} e^{-m_{f} s}\left[\mathcal{H}_{f}^{i} s \operatorname{coth}\left(\mathcal{H}_{f}^{i} s\right)-1-\frac{1}{3}\left(\mathcal{H}_{f}^{i}\right)^{2} s^{2}\right] .
\end{gathered}
$$

Calculation of this integral can be done by using the $\mathrm{\Gamma}-$ and $\zeta$-functions:

$$
\begin{aligned}
& \int_{0}^{\infty} t^{x-1} e^{-\alpha t} d t=\alpha^{-x} \Gamma(x), \quad \operatorname{Re} x>0, \quad \operatorname{Re} \alpha>0 \\
& \quad \int_{0}^{\infty} t^{s-1} e^{-v t}\left(1-e^{-t}\right)^{-1} d t=\Gamma(s) \zeta(s, v),
\end{aligned}
$$

$$
\text { Res }>0, \quad \operatorname{Rev}>0 \text {. }
$$

Let us set in denominator of (19) $s^{3} \rightarrow s^{3-\varepsilon}$ and consider the limit $\varepsilon \rightarrow 0$. We present (19) as the sum of four integrals and rewrite them using (20). In this way we get

$$
\begin{gathered}
8 \pi^{2} V_{q \cdot v a c, i, f}^{(1)}\left(H, H_{3}, H_{8}\right)= \\
-\left(2 \mathcal{H}_{f}^{i}\right)^{2}\left(\frac{m_{f}^{2}}{2 \mathcal{H}_{f}^{i}}\right)^{2-\varepsilon} \Gamma(\varepsilon-2) \\
-\frac{\left(\mathcal{H}_{f}^{i}\right)^{2}}{3}\left(\frac{m_{f}^{2}}{2 \mathcal{H}_{f}^{i}}\right)^{-\varepsilon} \Gamma(\varepsilon) \\
+2\left(\mathcal{H}_{f}^{i}\right)^{2} \zeta\left(\varepsilon-1 ; \frac{m_{f}^{2}}{2 \mathcal{H}_{f}^{i}}\right) \Gamma(\varepsilon-1) \\
+2\left(\mathcal{H}_{f}^{i}\right)^{2} \zeta\left(\varepsilon-1 ; \frac{m_{f}^{2}}{2 \mathcal{H}_{f}^{i}}+1\right) \Gamma(\varepsilon-1) .
\end{gathered}
$$

Then we make an expansion in series over $\varepsilon$ and use $\Gamma_{1}$-function for calculation of the derivative

$$
\begin{gathered}
\zeta^{\prime}(-1 ; x)=\left.\frac{d \zeta(\varepsilon-1, x)}{d \varepsilon}\right|_{\varepsilon=0}=\Gamma_{1}(x)+\zeta^{\prime}(-1), \\
\Gamma_{1}(x)=\int_{0}^{x} \ln \Gamma(y) d y+\frac{1}{2} x(x-1)-\frac{1}{2} x \ln (2 \pi) .
\end{gathered}
$$

As a result, we obtain Eq.(16) for vacuum energy.

\section{Appendix B}

The general method for calculation of the high temperature asymptotic used in Secs. 2-3 has been developed in [1, 19.

For the high temperature expansion of the one-loop quark effective potential we perform the following steps. First, the expression in the brackets in (6) we expand in series over $s$ near the point $s=0$ and insert into the integral over s. First two terms of interest are

$$
\begin{gathered}
4 \pi^{2} V_{i, f}=\sum_{l=1}^{\infty}(-1)^{l} \\
\times \int_{0}^{\infty} d s e^{-m_{f}^{2} s-\frac{\beta^{2} l^{2}}{4 s}}\left(\frac{\left(\mathcal{H}_{f}^{i}\right)^{2}}{3 s}-\frac{1}{45}\left(\mathcal{H}_{f}^{i}\right)^{4} s\right) .
\end{gathered}
$$

Then we integrate over $s$ by means of the well-known formula for K-function

$$
\int_{0}^{\infty} d s s^{n-1} e^{-a s-\frac{b}{s}}=2\left(\frac{a}{b}\right)^{\frac{n}{2}} K_{n}(2 \sqrt{a b}) .
$$

As a result, we can separate the sum over $l$ from the fields $\mathcal{H}_{f}^{i}$

$$
\begin{aligned}
& 4 \pi^{2} V_{i, f}=\frac{2}{3}\left(\mathcal{H}_{f}^{i}\right)^{2} \sum_{l=1}^{\infty}(-1)^{l} K_{0}\left(m_{f} \beta l\right) \\
& -\frac{\left(\mathcal{H}_{f}^{i}\right)^{4}}{90 m_{f}^{4}} \sum_{l=1}^{\infty}(-1)^{l}\left(m_{f} \beta l\right)^{2} K_{2}\left(m_{f} \beta l\right) .
\end{aligned}
$$

For the next sums, we can calculate the asymptotic expressions by using Mellin's transformation [11, 19]

$$
\begin{gathered}
\sum_{n=1}^{\infty}(-1)^{n} K_{0}(\omega n)=\frac{1}{2}\left(\gamma+\ln \left(\frac{\omega}{\pi}\right)\right) \\
+\sum_{n=1}^{\infty} \frac{\left(2^{2 n+1}-1\right) \omega^{2 n} \zeta^{\prime}(-2 n)}{2^{2 n}(n !)^{2}} \\
\quad \sum_{n=1}^{\infty}(-1)^{n}(\omega n)^{2} K_{2}(\omega n)=-1 \\
+\sum_{n=2}^{\infty}\left(\frac{\omega}{2}\right)^{2 n}\left(2^{2 n+1}-1\right) \frac{4}{n !(n-2) !} \zeta^{\prime}(-2 n) .
\end{gathered}
$$

As a result, we obtain the high temperature expansion of the effective potential (17). The high temperature expansion of the gluon one-loop effective potential and daisies has been done in [10]. 


\section{References}

[1] H. Satz, vol. 841 (2012) of Lecture Notes in Physics. Springer.

[2] J. Greensite, vol. 821 (2011) of Lecture Notes in Physics. Springer.

[3] O. K. Kalashnikov, Fortsch. Phys. 32 (1984) 525.

[4] G. S. Bali et al., PoS, ConfinementX:197, 2012.

[5] L. Levkova, C. DeTar, Phys. Rev. Lett. 112(1) (2014) 012002.

[6] K. Szabo, PoS, LATTICE2013 (2014) 014.

[7] G. S. Bali, F. Bruckmann, G. Endrödi, A. Schäfer, PoS, LATTICE2013 (2014) 182.

[8] D. Grasso H. R. Rubinstein, Phys. Rept. 348 (2001) 163-266.

[9] E. Elizalde, V. Skalozub, Eur. Phys. J. C 72 (2012) 1968.

[10] V.V. Skalozub, A.V. Strelchenko, Eur. Phys. J. C 33 (2004) 105.

[11] V. Skalozub, Int. J. Mod. Phys. A 11 (1996) 5643.

[12] V. Skalozub, M. Bordag, Nucl. Phys. B 576 (2000) 430.

[13] M.D. Pollock, Int. J. Mod. Phys. D 12 (2003) 1289.
[14] V.I. Demchik, V.V. Skalozub, Eur. Phys. J. C 27 (2003) 601.

[15] J. I. Kapusta, Finite-temperature Field Theory. Cambridge University Press, 1989.

[16] V. Demchik, V. Skalozub, J. Phys. A 41 (2008) 16405 .

[17] A.I. Akhiezer, V.B. Berestetski. Kvantovaya electtrodinamika. "Nauka", Moscow, 624 p., 1969.

[18] S. Antropov, M. Bordag, V. Demchik and V. Skalozub, Intern. J. Mod. Phys. A 26 (2011) 4831.

[19] H. E. Haber, H. A. Weldon, J. Math. Phys. 23 (1982) 1852.

[20] V. Demchik, V. Skalozub, Phys. Part. Nucl. 46(1) (2015) 1-23.

[21] V. Skalozub, P. Minaev. Visnyk Dnipropetrovs'kogo univ. Fizuka. Radioelectronika, 24: 25, 2016. arXiv:1612.00216, hep-ph].

[22] A.O. Starinets, A. S. Vshivtsev, V. Ch. Zhukovsky, Phys. Lett. B 322 (1994) 403.

[23] N.O. Agasian, S. M. Fedorov, Phys. Lett. B 663 (2008) 445.

[24] P. Cea, L. Cosmai, M. D’Elia, JHEP. 0712 (2007) 097.

[25] G. S. Bali et al. JHEP , 1202 (2012) 044. 